\title{
An Integral Probe for Capillary Zone Electrophoresis/Continuous-Flow Fast Atom Bombardment Mass Spectrometry
}

\author{
M. J.-F. Suter* and R. M. Caprioli \\ The Analytical Chemistry Center and Department of Biochemistry and Molecular Biology, The University of \\ Texas Medical School, Houston, Texas, USA
}

\begin{abstract}
An integral probe for capillary zone electrophoresis/continuous-flow fast atom bombardment mass spectrometry was constructed and operated in either the coaxial or liquid-junction interface mode. Results using these interfaces for the analyses of synthetic peptides are presented. The coaxial arrangement attains the best electrophoretic performance, generally providing a greater number of theoretical plates. However, in that the electrophoresis times are generally greater for the liquid-junction interface because there is no mechanical flow resulting from the source vacuum, the overall separation efficiency of the liquid-junction interface is equal to or greater than that of the coaxial interface. In addition, the liquid-junction interface is easier to set up and operate, and allows larger inner diameter capillaries to be used to achieve higher sample loads. (J Am Soc Mass Spectrom 1992, 3, 198-206)
\end{abstract}

C apillary zone electrophoresis (CZE) is based on early experiments reported by Hjertén [1], performed in a rotating $3-\mathrm{mm}$ ID quartz tube. Although Virtanen [2] and Mikkers et al. [3] showed the advantages of using capillaries with smaller inner diameters, a major breakthrough occurred when Jorgenson and Lukacs [4] obtained high resolution separations.

CZE is becoming an important tool in biochemistry, especially because the very high numbers of theoretical plates that can be obtained can provide high resolution separations [5]. Often the separation selectivity of CZE is complementary to that of high performance liquid chromatography (HPLC) [6]. The small volume of sample required for CZE (picoliters to nanoliters) has enabled, for example, direct sampling from single cells [7]. Furthermore, it has been shown that the biological activity of samples is not affected by this technique [8].

Separations arise because of different electrophoretic mobilities $\left(\mu_{\mathrm{ep}}\right)$ of analytes. The mobilities can be manipulated by adjusting the $\mathrm{pH}$ of a chosen electrolyte [6]. The very high number of theoretical plates reached in $\mathrm{CZE}$ is a consequence of the fact that the major factor contributing to zone broadening is axial diffusion as described by the Stokes-Einstein law [9],

*Present address: Swiss Federal Institute for Water Resources and Water Pollution Control (EAWAG), CH-8600 Dübendouf, Switzerland.

Address reprint requests to Richard M. Caprioli, The Analytical Chemistry Center and Department of Biochemistry and Molecular Biology, University of Texas Medical School, Houston, TX 77030 . assuming ideal sample injection, and minimal wall interactions and Joule heating. Thus short capillaries and a high electric field should lead to excellent system performance. It is noted that the measurement of the number of theoretical plates of a system is only one measure of performance. For any given sample, the separation efficiency of a system, i.e., the ability of a given electrophoresis experiment to physically separate two components in the sample mixture, is the most important criterion. Here, the time period and buffer properties of the electrophoresis process is most important. Thus, it is possible to have a system give a greater number of theoretical plates than another, but to show poorer separation efficiency of a given sample.

In CZE, unlike in pressure-driven systems with their parabolic flow profile, the injected sample volume or migrating zone is not distorted because the electroosmotic flow shows a pluglike velocity distribution. The magnitude of this velocity $\left(v_{\text {eo }}\right)$ depends on the applied CZE voltage and the $\xi$-potential, the voltage across the solvent/solute layer adjacent to the inner wall of the capillary. This $\xi$-potential is affected by the $\mathrm{pH}$ and ionic strength of the electrolyte $[10,11]$ and can be further manipulated by coating or modifying the fused silica $[12,13]$ or by applying an external field [14]. Solvated positively charged ions move toward the more negatively charged electrode, providing a net mechanical flow of liquid, typically of the order of nanoliters per minute. This is termed electroosmotic flow. Migration of charged species, i.e., positive compounds toward the negative electrode 
and negative compounds toward the positive electrode, termed electrophoretic mobility, occurs concurrently. If the electroosmotic mobility of the electrolyte is higher than the electrophoretic mobility of the charged species, all analytes, both positively and negatively charged, will elute at the cathodic end of the system.

Various detectors have been used in combination with $\mathrm{CZE}$, with mass spectrometry being unique for its combination of high sensitivity and mass specificity. The first coupling of CZE with mass spectrometry was reported by Olivares et al. [15], using an electrospray interface. More recently, the electrospray technique has been improved by the use of a sheath liquid [16]. Andresen and Fought [17] proposed an interface with double capillaries for various desorption techniques. A setup similar to the electrospray interface was described by Lee et al. [18] using an electrospray source incorporating pneumatically assisted nebulization (also called ion spray). The coupling of CZE with continuous-flow fast atom bombardment (CF-FAB) ionization [19] was first reported by Minard et al. [20]. Tomer and co-workers [21] described a coaxial interface for CF-FAB and capillary separation techniques, such as $\mathrm{CZE}$, which provides an efficient coupling of liquid flow techniques with mass spectrometers. A liquid-junction interface has been described by Caprioli et al. [22].

We have designed, constructed, and tested an integral CZE/CF-FAB probe that can utilize either a coaxial interface or a liquid-junction interface arrangement. This article describes the performance of this combination probe and compares results obtained using the two interfaces.

\section{Experimental}

\section{CZE Mass Spectrometry Interface}

The capability for the use of different interface operating modes was deemed important in the construction of an integral CZE/MS probe. The main advantage of the coaxial interface rests on the fact that it has virtually no dead volume because the CZE capillary terminates directly at the probe tip, as shown in Figure 1a. The electric field strength within the electrolyte is given with two well-defined potentials: the voltage applied to the anodic buffer container and the ion source potential, which acts as the cathodic counterpart. The liquid-junction interface, on the other hand, has significantly greater dead volume, but has the advantage that it is exceedingly easy to assemble and operate (Figure $1 \mathrm{~b}$ ) and efficiently decouples the vacuum-induced mechanical flow produced by the high vacuum ion source.

The diagrammatic representation of the CZE/CFFAB probe for a high resolution mass spectrometer is shown in Figure 2. For the coaxial setup, the CZE capillary (10 $\mu \mathrm{m}$ i.d./184 $\mu \mathrm{m}$ o.d., $711 \mathrm{~mm}$ long, coated on the outside with $10 \mu \mathrm{m}$ of polyimide; Polymicro Technologies, Inc., Phoenix, AZ) is guided coaxially within a sheath capillary (203 $\mu \mathrm{m}$ i.d./293 $\mu \mathrm{m}$ o.d., $\times 500-600 \mathrm{~mm}$ ), which extends from a plexiglass tee to the probe tip. The dead volume of this setup is $32 \mathrm{~nL}$, if the inner capillary is pulled back 1 $\mathrm{mm}$ from the end of the outer capillary. The CF-FAB matrix is fed into the sheath capillary at a rate of 2 $\mu \mathrm{L} / \mathrm{min}$ by a syringe pump (Brownlee Labs, Santa Clara, CA) connected to the interface "tee." The liquid-junction interface was operated as described earlier [22], using a $51-\mu \mathrm{m}$ i.d. $/ 144-\mu \mathrm{m}$ o.d., $\times 600$ 700-mm CZE capillary and a 540-mm-long CF-FAB capillary with an i.d. of $75 \mu \mathrm{m}$ and an o.d. of $260 \mu \mathrm{m}$ (Polymicro Technologies, Inc., Phoenix, AZ). The measured flow rate for the CF-FAB solvent was 8 $\mu \mathrm{L} / \mathrm{min}$. The dead volume given with the CF-FAB transfer line is $2.4 \mu \mathrm{L}$.

The apparatus is made of plexiglass and is attached to a standard stainless steel probe shaft that fits the introduction lock of the mass spectrometer. The high voltage (0-50 kV supply; model 205A-50P, Bertan Associates, Inc., Hicksville, NY) is applied to the buffer and sample container via a shielded cable ending in 0.5-mm o.d. platinum wire and is controlled by a safety interlock switch mounted on the outside of the probe. A plexiglass handle that is guided through a 1/4-in. fitting with teflon ferrules in the lid of the probe allows placement of the capillary in either the buffer or sample container. The CZE capillary is held in place by a conventional 3/8-in. gas chromatograph injector septum.

Adjustment of the CF-FAB (outer) capillary end in or out of the plane of the target surface may be desirable under operating conditions, and so a position adjustment device was developed. With this setup, the sheath capillary is held in place by a graphite ferrule in a 1/16-in. stainless steel fitting that is soldered onto a support flange. The whole flange can be pushed back by a nut, turning on another fitting through which is passed the stainless steel inner tube of the probe shaft. This allows for easy and precise positioning of the capillary in the probe tip.

The CZE capillaries were preconditioned with 10 column volumes of $0.1 \mathrm{M} \mathrm{KOH}$ and rinsed with 18 $\mathrm{M} \Omega$ water. For low $\mathrm{pH}$ operation the capillary was conditioned with an additional wash with $0.1 \mathrm{M} \mathrm{HCl}$ before equilibration with the desired buffer [5].

\section{Mass Spectrometry}

CF-FAB mass spectrometry was performed using a Finnigan-MAT (San Jose, CA) model 90 double-focusing mass spectrometer. The instrument was typically operated at full acceleration voltage of $4.7 \mathrm{kV}$, a resolution of $\sim 1200$ and a scan speed of $5 \mathrm{~s}$ per decade. The scan range was $m / z \quad 500-660$ for the MRFA/FLEEL experiments and 1200-1750 for the synthetic peptides. The FAB gun was operated at 8 


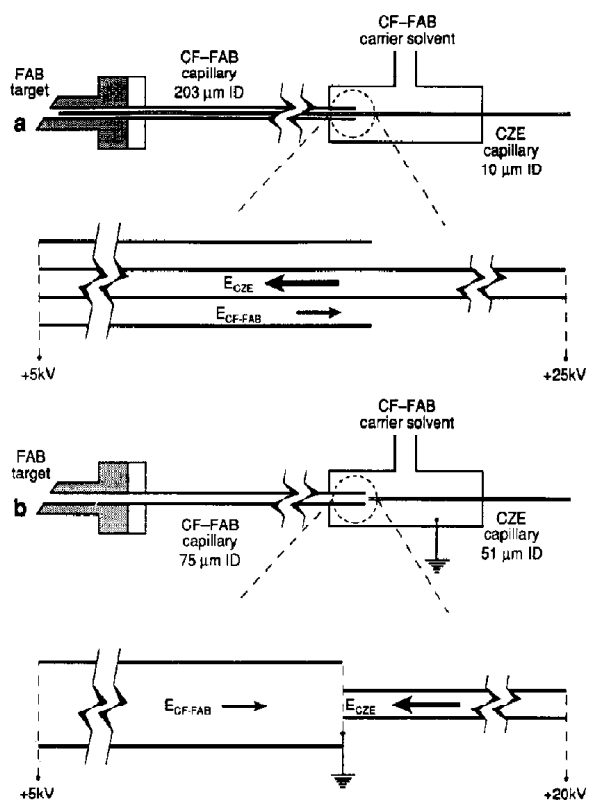

Figure 1. Schematic representations of the coaxial (a) and liquid-junction interfaces (b). The arrows indicate the orientation and magnitude of the electric field strength $E$ (see text for details). $E_{\text {CF-FAB }}$ in a is defined by the source potential ( $+5 \mathrm{kV}$ ) and the grounded syringe pump (not shown).
$\mathrm{kV}$ with xenon gas to give a monitor current of -40 $\mu \mathrm{A}$. The ion source pressure was normally $1.5 \cdot 10^{-4}$ mbar with the coaxial interface and $1.7-3.0 \cdot 10^{-4}$ mbar with the liquid-junction interface. The ion source temperature was set to about $45^{\circ} \mathrm{C}$. The flow rate of the CF-FAB solution was $\sim 2 \mu \mathrm{L} / \mathrm{min}$ for the coaxial interface and $8 \mu \mathrm{L} / \mathrm{min}$ for the liquid-junction interface.

\section{Chemicals}

All solvents were HPLC grade. The water had a resistance of $18 \mathrm{M} \Omega$. The peptide MRFA was obtained from Research Plus, Inc. (Bayonne, NJ), and FLEEL, from Vega Biochemicals (Tucson, AZ). Synthetic peptides were prepared by solid phase synthetic methods using an Applied Biosystems Inc. (Foster City, CA) model $430 \mathrm{~A}$ automated peptide synthesizer. Mesityl oxide was obtained from Aldrich Chemical Company, Inc. (Milwaukee, WI). All other chemicals were of analytical grade or better.

The CZE buffer system was a $40 \mathrm{mM}$ citric acid solution adjusted with $\mathrm{NH}_{4} \mathrm{OH}$ to $\mathrm{pH} 3.0$ for the MRFA/FLEEL mixture and $\mathrm{pH} 2.5$ for the synthetic peptides. This system has been previously used with good results [22]. The composition of the CF-FAB solvent was $25 \%$ glycerol in $5 \mathrm{mM}$ aqueous heptafluorobutyric acid for the coaxial interface and $5 \%$ glycerol $/ 3 \%$ acetonitrile in aqueous $6 \mathrm{mM}$ acetic acid $(\mathrm{pH}$ 3.4) for the liquid-junction interface.

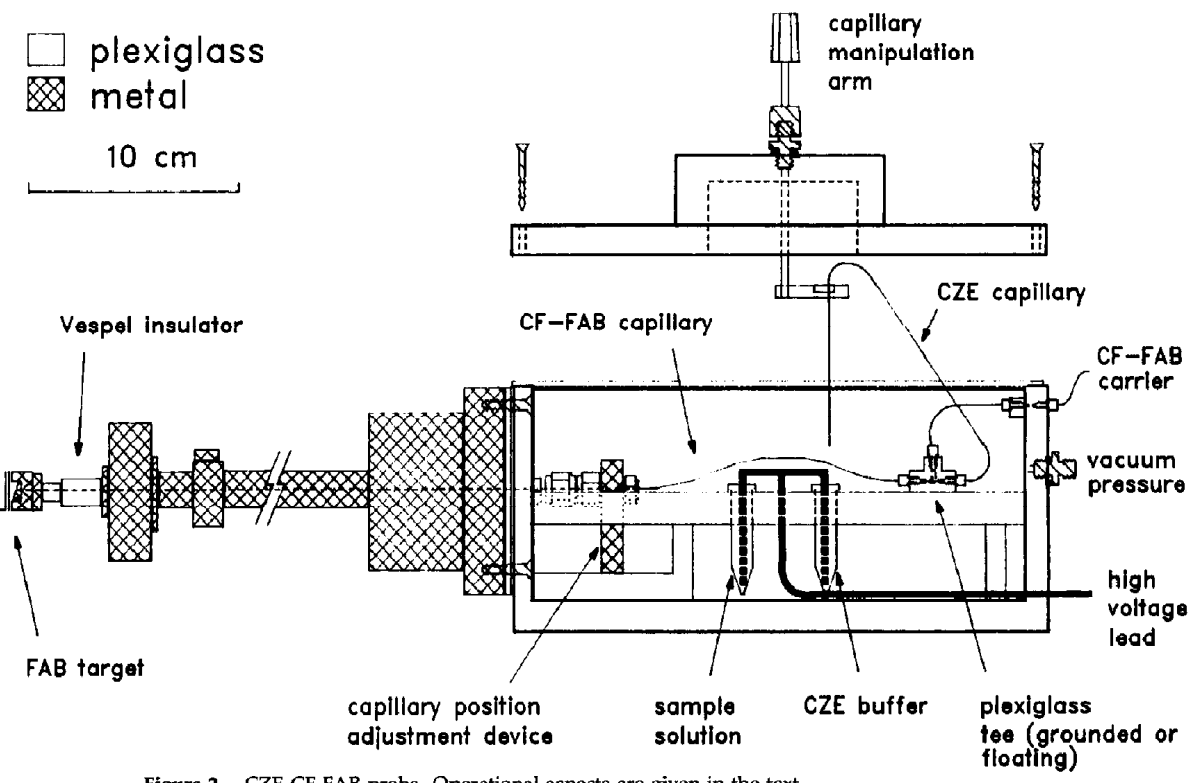

Figure 2. CZE CF-FAB probe. Operational aspects are given in the text 
All aqueous solvents and buffers were filtered using disposable Nalgene filter funnels $(0.45-\mu \mathrm{m}$ cellulose nitrate membrane, Nalge Co., Rochester, NY). Sample solutions were filtered using microfuge 0.45$\mu \mathrm{m}$ Durapore filter units (Millipore Corp., Bedford, MA).

\section{Results}

\section{Sample Loading}

Usually, for CZE employing ultraviolet (UV) or other non-mass spectrometric detectors, samples may be loaded into the CZE capillary by either electrokinetic or hydrodynamic methods. However, since in CZE/ CF-FAB mass spectrometry one end of the capillary (or connected transfer line) is in a high vacuum environment, some special considerations apply.

With the coaxial interface, sample injection is accomplished by making use of the pressure differential between the ends of the capillary (hydrodynamic injection; no voltage applied to the sample mixture). The pressure-induced flow rate can be determined with control runs where the electric field strength within the CZE capillary is zero, i.e., a voltage equivalent to the accelerating voltage of the mass spectrometer is applied to the anodic buffer compartment. The pressure-induced flow, and thus the sample load, can easily be calculated from the resulting elution time and typically is on the order of $-3 \mathrm{~nL} / \mathrm{min}$. When electrokinetic injection is used while the buffer and sample compartment are at atmospheric pressure, vacuum-induced hydrodynamic sample loading still occurs because the target tip is in the high vacuum source. Reducing the pressure in the buffer and sample compartment can minimize this vacuum-induced flow, although this requires the use of solutions of low volatility to avoid concentration changes due to evaporation of the sample solvent and for this reason has not been further investigated.

In the case of the liquid-junction interface, sample injection was accomplished either hydrodyramically by drawing on a syringe connected to the interface compartment (as described elsewhere [22]), or electrokinetically. In the latter, the sample load $Q$ is calculated according to

$$
Q=\left(\mu_{\mathrm{eo}}+\mu_{\mathrm{ep}}\right) \cdot V C t \pi r^{2} / L
$$

where $\mu_{\mathrm{eo}}$ is the electroosmotic mobility, $\mu_{\mathrm{ep}}$ the electrophoretic mobility, $V$ is the applied CZE voltage, $C$ the sample concentration, $t$ the injection time, $r$ the inner radius of the CZE capillary, and $L$ the capillary length [4]. For most of the work in this study, only the electrokinetic injection method was used with the liquid-junction interface because it gave the sharpest peaks. Hydrodynamic sample loading tends to cause more of a diffuse sample zone because of the parabolic flow profile resulting from the applied pressure. Discrimination of the more negatively charged species that can occur when using electrokinetic injection was considered of minor consequence for the samples used in this work, although it can be significant in some cases.

The CZE/CF-FAB mass spectrometry combination using either the coaxial or liquid-junction interface differs from conventional CZE detector arrangements in that the cathodic end of the capillary is surrounded by CF-FAB solvent rather than CZE buffer. For positive ion FAB analysis, this cathode is at the accelerating potential of the mass spectrometer. Negatively charged matrix components in the interface reservoir can enter the CZE capillary at the interface end by electrophoretic migration and diffusion, although the electroosmotic flow tends to minimize this. This can give rise to concentration and $\mathrm{pH}$ gradients depending on the electroosmotic flow, the buffer and the CF-FAB solvent used. Although this effect is usually small, it may have an effect on overall performance in some cases.

Under the experimental conditions described, the maximal sample volume, if one takes a $1 \%$ sample plug relative to the capillary volume as the upper limit [23], is $560 \mathrm{pL}$ for the coaxial interface and $14.5 \mathrm{~nL}$ for the liquid-junction interface.

\section{Interface Parameters}

Although the coaxial interface can provide sharper peak profiles, it has several disadvantages: (1) it is prone to mechanical flow within the CZE capillary due to the pressure drop between the anodic buffer compartment at atmospheric pressure and the cathodic end in the high vacuum of the mass spectrometer, generally having a deleterious effect on separating efficiency; (2) the very small diameter capillaries usually employed makes on-line UV detection problematic and are easily plugged; and (3) it is more difficult to set up because the CZE capillary has a thinner wall and needs to be inserted coaxially into the CF-FAB capillary, often damaging one or both capillaries. Although high voltage discharge through the wall can occur with both interfaces, this can be minimized by avoiding contact (or near-contact) of the capillary to any ground point. The major disadvantage of the liquid-junction interface is the band broadening that occurs in the CF-FAB capillary. This effect is discussed below in more detail.

With both interfaces, the CF-FAB solvent has to be a sufficiently good electrolyte to provide electrical contact between the cathodic end of the CZE capillary and the cathode potential, whether this is defined by a high voltage source or instrument ground. This arrangement results in a small electric field within the CF-FAB solvent as shown in Figure 1, defined by the accelerating potential of the mass spectrometer and the grounded interface or associated syringe pump. Thus in the case of the coaxial setup, this field adds to the $\zeta$ potential across the capillary wall, and has an 
increasing effect on the electroosmotic mobility toward the plexiglass interface. There is an analogous effect for the liquid-junction interface, as shown in Figure 1. However, in most cases, this effect should be small because the field strength is low $(87 \mathrm{~V} / \mathrm{cm})$ and the analytes are transported at a flow rate of $\sim 8$ $\mu \mathrm{L} / \mathrm{min}$ and consequently will be exposed to the freld for only $\sim 15-20 \mathrm{~s}$.

One of the advantages of adding make-up solvent such as CF-FAB carrier solution is that it allows one to run with higher buffer concentrations, because the CZE buffer will be diluted with the CF-FAB solvent. For example, even with $20 \mathrm{mM} \mathrm{NaCl}$ solutions in the CZE buffer, tryptic fragments rarely show sodium adduct ions in the FAB mass spectra. However, a disadvantage is the dilution and band-broadening effect of this mixing. This effect is greatest with the liquid-junction interface because it has a significant dead volume $(2.4 \mu \mathrm{L})$ in the capillary connecting the plexiglass junction with the probe tip.

Joule heating which can cause a radial temperature gradient leading to mobility changes is within reasonable limits in the apparatus described in this report because the capillaries used have inner diameters that favor good heat dissipation $(<80 \mu \mathrm{m})$ [10]. In addition, the capillaries are cooled for $70 \%$ (liquid-junction interface) to $85 \%$ (coaxial interface) of their length.
In the case of the coaxial interface, the cooling is achieved with the surrounding CF-FAB matrix within the coaxial setup from the plexiglass tee to the probe tip. For the liquid-junction interface, the part of the capillary from the anode to the UV detector is in a plexiglass box which is kept at room temperature.

\section{Electrophoretic Performance}

A test mixture consisting of two peptides, Met-ArgPhe-Ala (MRFA; $[\mathrm{M}+\mathrm{H}]^{+}=m / z$ 524.2) and PheLeu-Glu-Glu-Leu (FLEEL; $[\mathrm{M}+\mathrm{H}]^{+}=m / z$ 650.3) was used to establish the operating performance of the probe device shown in Figure 2. The peptides were dissolved in water and $0.3 \%$ trifluoroacetic acid to give final concentrations of $\sim 1 \mathrm{mM}$. By dissolving the peptides in water rather than buffer, the sample zones will be compressed at the beginning of the CZE run $(\mathrm{H}$. H. Lauer, personal communication). Figure 3 shows the total ion recordings for this test mixture, obtained using the coaxial and the liquid-junction interfaces. With the coaxial interface, $325 \mathrm{fmol}$ of MRFA were detected with a signal-to-noise ratio of 30:1 and a peak width measured at half height of $7.4 \pm 1.1 \mathrm{~s}(n=8)$. With the liquid-junction interface, $275 \mathrm{fmol}$ of MRFA were detected with a signalto-noise ratio of $15: 1$ and a peak width of $36 \pm 10 \mathrm{~s}$

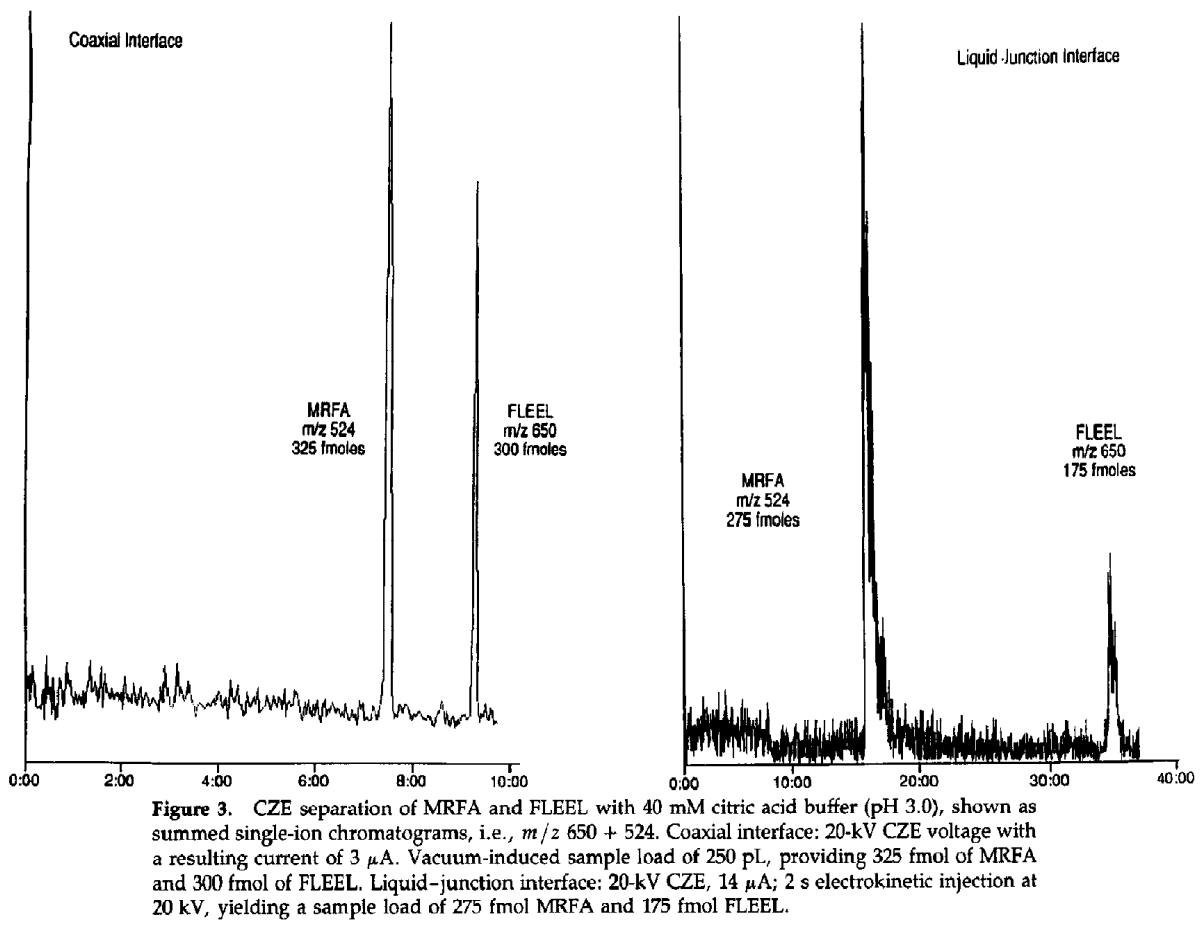


$(n=4)$. The liquid-junction interface gives rise to more zone broadening than does the coaxial interface, as seen in Figure 3. It should be noted that the different relative intensities of FLEEL and MRFA in the two panels in Figure 3 is the result of the sample loading method, not the interface. The electrokinetic sample loading used with the liquid-junction interface shows discrimination toward the more negatively charged peptide FLEEL, while the sample load resulting from pressure-induced injection in case of the coaxial interface reflects the truer initial sample concentrations.

The number of theoretical plates calculated for the peptide separations shown in Figure 3 is given in Table 1 . The longer migration times obtained with the liquid-junction interface and the relatively large dead volume contribute to analyte zone broadening. The latter effect is apparent in Table 1 from the UV data for the liquid-junction interface showing only a slightly reduced CZE performance compared to the coaxial interface, but a significantly increased bandwidth at the mass spectrometer source. On the other hand, the separation efficiency is much better with the liquid-junction interface. The elution time for MRFA was $944 \mathrm{~s}(\sigma=29, n=7)$, which was an average of $1107 \mathrm{~s}$ shorter than that for FLEEL in case of the liquid-junction interface. For the coaxial interface the retention times were $t_{\mathrm{e}}$ (MRFA) $=412 \mathrm{~s}(\sigma=34$, $n=10)$ and the difference in retention time $\Delta t_{\mathrm{e}}=92$ $s(\sigma=17, n=10)$. This increased efficiency for the liquid-junction interface is shown in Figure 3 . It is presumably a major consequence of the greater time period during which electrophoretic separation occurs.

Even though all CZE experiments were done at a $\mathrm{pH}$ that gives rise to few charged silanol groups on

Table 1. Number of theoretical plates $(N)^{\mathrm{a}}$

\begin{tabular}{lccccc} 
& \multicolumn{2}{c}{ Cosial interface } & & \multicolumn{2}{c}{ Liquid-junction interface } \\
\cline { 2 - 3 } \cline { 5 - 6 } Species & UV $^{b}$ & Mass & & & Mass \\
spectrometry & & UV $^{c}$ & spectrometry \\
\hline \hline MRFA & NA & 23,000 & & 19,000 & 2,000 \\
FLEEL & NA & 70,000 & & 48,000 & 13,000 \\
\hline
\end{tabular}

${ }^{\mathrm{a}} N=5.54 \cdot\left(t_{\theta} / w_{1 / 2}\right)^{2} ; t_{\theta}$ equals the elution time and $w_{1 / 2}$ the peak width at half height.

${ }^{b}$ UV data are not available (NA) for the coaxial interface.

"The UV window is at a distance of $511 \mathrm{~mm}$ from the anode, prior to the liquid-junction interface. the capillary inner surface and the analyte concentrations were kept low, effects of wall interactions were observed with both interfaces. MRFA, which carries the higher positive charge, generally produced broader peaks. Wall coatings, which can lead to significant increases in efficiencies, were deliberately avoided in this work to achieve valid comparisons and to allow determination of the magnitude of these effects.

\section{Coaxial Interface}

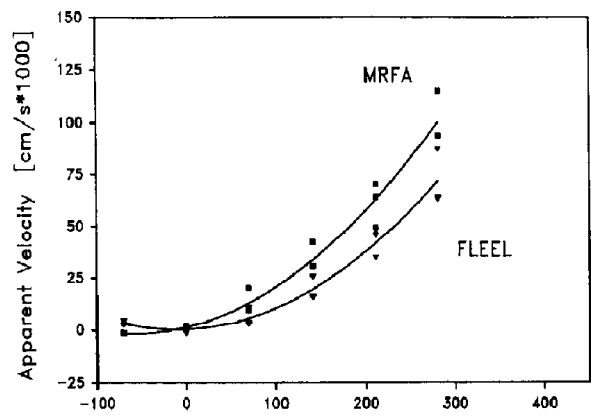

Liquid-Junction Interface

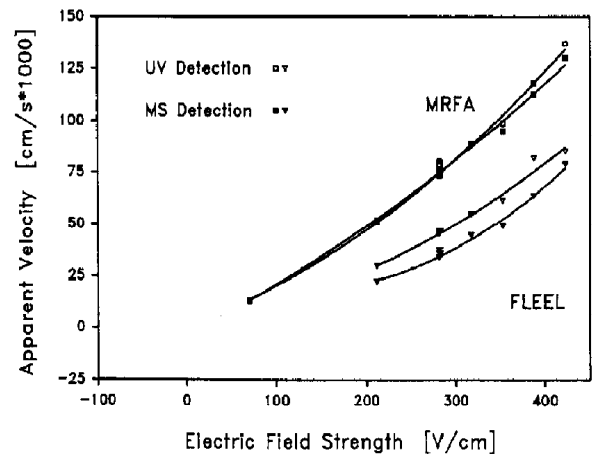

Figure 4. Apparent velocity vapp versus electric field strength $E$. Squares, MRFA; triangles, FLEEL (filled symbols represent detection by mass spectrometry and open symbols detection at the UV window). Experimental conditions as in Figure 3.

Table 2. Apparent velocity and charge for MRFA and FLEEL with the liquid-junction interface

\begin{tabular}{|c|c|c|c|c|}
\hline \multirow[b]{2}{*}{ Species } & \multicolumn{2}{|c|}{ Charge } & \multicolumn{2}{|c|}{$v_{\text {app }} \cdot 10^{-3}[\mathrm{~cm} / \mathrm{s}](E=281 \mathrm{~V} / \mathrm{cm})$} \\
\hline & $\mathrm{pH} 3.0$ & pH 3.4 & $U^{8}{ }^{8}$ & $\mathrm{MS}^{\mathrm{b}}$ \\
\hline $\begin{array}{l}\text { MRFA } \\
\text { FLEEL }\end{array}$ & $\begin{array}{l}1.18 \\
0.09\end{array}$ & $\begin{array}{r}1.08 \\
-0.14\end{array}$ & $\begin{array}{l}74.3 \pm 2.2 \\
45.0 \pm 1.5\end{array}$ & $\begin{array}{l}76.1 \pm 2.8 \\
35.0 \pm 2.1^{c}\end{array}$ \\
\hline
\end{tabular}

"UV detection; $511 \mathrm{~mm}$ from anode compartment.

${ }^{b}$ Mass spectrometric detection; total CZE caplllary length $(711 \mathrm{~mm})$, elution time corrected for $15 \mathrm{~s}$ spent in the CF-FAB capillary.

${ }^{c}$ Number of experiments; $n=5$ for this value, elsewhere $n=7$. 
The apparent velocity of an electrolyte through the CZE capillary is the sum of the electroosmotic $\left(v_{\mathrm{eo}}\right)$ and electrophoretic velocity $\left(v_{\mathrm{ep}}\right)$, corrected for pressure-induced flow ( $v_{\Delta p}$, equal to zero for the liquid-junction interface, if the time spent in the CF-FAB capillary is neglected) [1, 9]:

$$
v_{\text {app }}=v_{\text {eo }}+v_{\text {ep }}-v_{\Delta \mathrm{p}}=\left(\mu_{\mathrm{eo}}+\mu_{\mathrm{ep}}\right) \cdot E-v_{\Delta \mathrm{p}}
$$

In the absense of thermal gradients due to Joule heating, one would expect a linear relationship between $v_{\text {app }}$ and $E$. In Figure 4 both interfaces show a deviation from a straight line. Note that for the coaxial interface values for an electric field strength $>281$ $\mathrm{V} / \mathrm{cm}$ are not reported. Although higher voltages can be applied, the resulting spectra are generally noisy, due to arcing through the CZE capillary. The data at negative value $(-70.3 \mathrm{~V} / \mathrm{cm})$ are obtained when no voltage is applied, but the source potential is still at $+5 \mathrm{kV}$. All values are corrected for $v_{\Delta \mathrm{p}}$. Higher voltage can be used with the liquid-junction interface, due to its thicker capillary wall. However, data collection is slow at low electric field strength $\left[t_{\mathrm{e}}\right.$ (MRFA) $=$ $90 \mathrm{~min}$ at $E=70.3 \mathrm{~V} / \mathrm{cm}]$ and once the absorbent pads on the probe tip were saturated with glycerol, stability in the ion source decreased rapidly. For the coaxial interface, part of the deviation from a straight line is due to the fact that in addition to decreased viscosity with higher freld strength, caused by Joule heating, $v_{\Delta p}$ was measured at $E=0$ and the change in viscosity with varying field strength was not taken into account for the correction of the various data points. Note that in the case of the coaxial interface, the apparent velocity of FLEEL is even positive at negative electric field strength $\left[v_{\text {app }}\right.$ (FLEEL) $>0$ at $E$ $=-70.3 \mathrm{~V} / \mathrm{cm}$ ]. The curves for the liquid-junction interface show that the overall effect of the interaction of the CF-FAB matrix solution with the sample in the CZE capillary is significant. The separation efficiency is higher compared to the coaxial interface, as described earlier, because of the longer electrophoresis times. Also, the values for $v_{\text {app }}$ (FLEEL) calculated from the UV traces differ clearly from those obtained with the mass spectrometer for the same analysis, whereas $v_{\text {app }}$ (MRFA) has similar values at both detection windows. Table 2 shows the respective values for $v_{\text {app }}$ obtained with the liquid-junction interface resulting from an electric field strength of $281 \mathrm{~V} / \mathrm{cm}$. Also listed are the calculated charges at $\mathrm{pH} 3.0$ (electrolyte at anode compartment) and $\mathrm{pH} 3.4$ (CF-FAB matrix at the liquid junction) (PIAA computer program kindly supplied by Applied Biosystems, Inc.). MRFA has a charge of $\sim 1$, while FLEEL, on the other hand, changes from a positively to negatively charged species. The values in Table 2 represent average velocities. The bulk velocity in the CZE capillary (average electroosmotic velocity) can be expected to be constant along the length of the capillary. The bulk flow rate was measured with the neutral marker mesityl oxide at an electric field strength of $281 \mathrm{~V} / \mathrm{cm}$ and was found to be $27.8 \cdot 10^{-3} \mathrm{~cm} / \mathrm{s}$. With this value and the average velocities from Table 2, the actual electrophoretic velocity of FLEEL along the capillary can be calculated. With the assumption of a linear change in electrophoretic velocity along the length of the capillary, one gets a velocity for FLEEL of $-8.4 \cdot 10^{-3}$ $\mathrm{cm} / \mathrm{s}$ at the UV detection window and $-28.4 \cdot 10^{-3}$ $\mathrm{cm} / \mathrm{s}$ at the liquid junction, a value close to the bulk velocity. ${ }^{1}$ This strongly suggests that the discontinuous electrolyte composition, possibly giving a signifcant $\mathrm{pH}$ gradient, is in part responsible for the increased elution time of FLEEL, relative to MRFA, when compared to the coaxial interface. Thus, the use of a discontinuous electrolyte composition can have a significant effect on the mobilities of the analytes and the overall separation efficiency.

An important test of these effects on the separation efficiencies of like compounds can be seen in the analyses of four synthetic peptides of closely related sequence as shown in Figure 5 . Even though peptides $\mathrm{A}\left([\mathrm{M}+\mathrm{H}]^{+}, m / z\right.$ 1425.8, G-KNGRILTL-RNPS $)$ and $\mathrm{B}\left([\mathrm{M}+\mathrm{H}]^{+}, m / z\right.$ 1522.9, G-KNGRILTLPRNPS) have an average charge of 3.34 , and $\mathrm{C}\left([\mathrm{M}+\mathrm{H}]^{+}, m / z\right.$ 1553.9, GKKNGRILTL-RNPS) and $\mathrm{D}\left([\mathrm{M}+\mathrm{H}]^{+}, \mathrm{m} / \mathrm{z}\right.$ 1650.9, GKKNGRILTLPRNPS), 4.34 at $\mathrm{pH} 2.5$, all four could be resolved to various extent with the system using either interface. The liquid-junction interface shows broader peaks and longer elution times, but shows better resolution of the compounds. The coaxial interface yields sharp peaks and higher sensitivity, but poor separation efficiency. Approximately $36 \mathrm{pmol}$ was close to the detection limit for the liquid-junction interface for these peptides, while this value was -1 pmol for the coaxial interface. The reason for the different detection limits for this sample is not known, nor was this difference observed for other peptide mixtures.

\section{Conclusion}

Comparing the two CZE/CF-FAB interfaces, the coaxial interface gives better performance with respect to the number of theoretical plates available. However,

\footnotetext{
${ }^{1}$ The linearity is a rough approximation, as $\mu_{\mathrm{ep}}$ has been shown to follow the empirical expression [24]:

$$
\mu_{\text {cF }}=D \cdot \ln (q+1) / n^{0.43}
$$

where $D$ is a proportionality constant, $q$ the charge of a molecule at a given $\mathrm{pH}$ (PIAA Computer Program), and $n$ its size. The correlation coefficient for the linear regression, relating $\operatorname{In}(q+1)$ to $\mathrm{pH}$ was calculated to be 0.996 over the range in question $(2.9<\mathrm{pH}<3.5$, calculated to be 0.996
$n=7$ ). Thus follows

$$
v_{\text {app }}(x)=v_{\text {app }}(0)-\alpha \cdot x
$$
}

where $v_{\text {app }}(0)$ is the initial apparent velocity and $\alpha$ a constant. The measured velocity $v_{\text {mea }}(x)=x / t$ represents the average velocity that can be written as

$$
v_{\text {mea }}(x)=\frac{1}{2}\left\{v_{\text {app }}(0)+v_{\text {app }}(x)\right\}=v_{\text {app }}(0)-\frac{1}{2} \alpha \cdot x
$$

With these two equations and the values for $v_{\text {mea }}(x)$ from Table 2 , the actual electrophoretic velocity $v_{\mathrm{ep}}(x)$ can be calculated. 

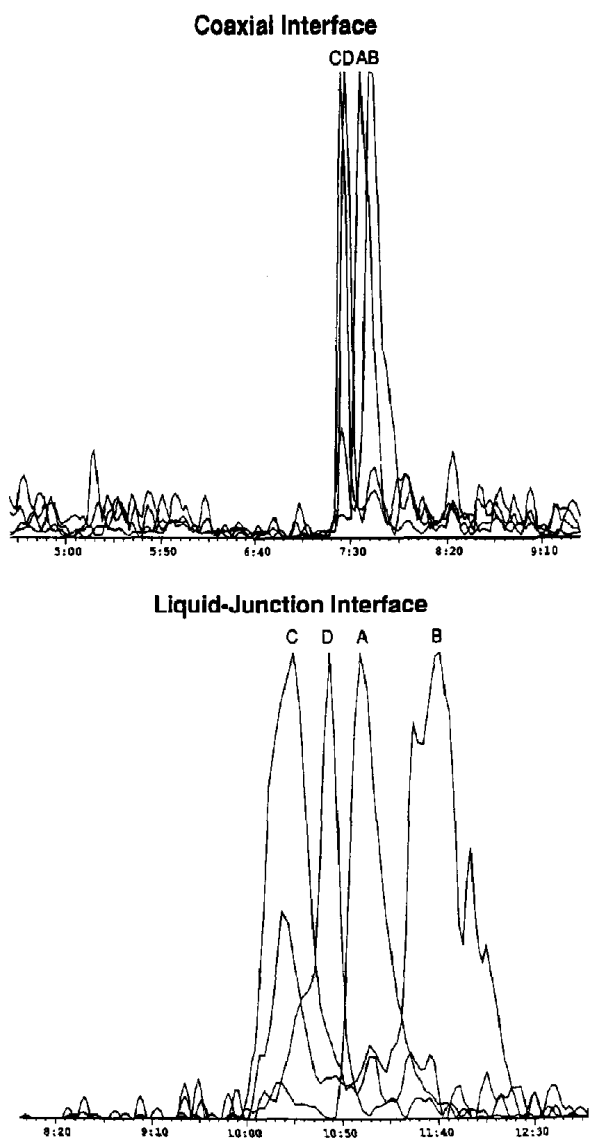

Figure 5. CZE separation of four synthetic peptides, with 40 $\mathrm{mM}$ citric acid buffer ( $\mathrm{pH} 2.5$ ), as shown with the overlaid reconstructed single ion chromatograms. CZE conditions: For the coaxial interface, conditions were the same as in Figure 3, except that the vacuum-induced sample load gave $840 \mathrm{fmol}$ of each peptide. With the liquid-junction interface, electrokinetic injection $(5 \mathrm{~s} / 20 \mathrm{kV})$ yielded an average sample load of 36 pmol.

the freld gradient in the CF-FAB solvent and the pressure-induced flow reduce overall $\mathrm{CZE}$ performance as measured by separation efficiencies. In addition, the coaxial interface is difficult to operate owing to the greater manipulation of relatively brittle capillaries and the risk of high voltage arcing through the thin capillary wall. The liquid-junction interface is easier to handle and set up, and gives better separation efficiencies, although it has the disadvantage of a significant dead volume and lower overall sensitivity. Also, a reversed electrophoretic effect in the CF-FAB capillary can lead to peak tailing and broader peaks, and CF-FAB matrix solution can affect the mobilities of the analytes.

Generally, a significant degree of CZE performance is lost in both interfaces. For mass spectrometric de- tection this is not necessarily a major problem because many mass spectrometers "scan" the mass range and require relatively broad peaks for optimal signal-tonoise recording. Thus, they cannot be properly used with electrophoretic peaks narrower in time than the scan time of the spectrometer. Generally, at least three scans per electrophoretic peak are desirable to provide minimal signal averaging. The reduced CZE resolution demanded by the use of a scanning mass spectrometer is compensated with the added mass specific information and potential for tandem mass spectrometry measurements. This, together with the complementary selectivity of CZE compared to HPLC, makes the CZE mass spectrometry combination an important new tool for the solution of analytical problems. Also, new mass spectrometer analyzer/detectors that have wide mass range signal integrating capabilities are becoming more widely available, e.g., ion traps, time-of-flight analyzers, and array detectors.

\section{Acknowledgment}

We thank Dr. W. T. Moore for the synthetic peptides shown in Figure 5. Support for this work was provided by the National Institutes of Health under grant 1-RO1-GM 43783-01.

\section{References}

1. Hjertén, S. Chromatog. Rev. 1967, 9, 122-219.

2. Virtanen, R. Acta Polytech. Scand. 1974, 123, 1-67.

3. Mikkers, F.; Everaerts, F.; Verheggen, T. J. Chromatogr. 1979, 169, 1-10.

4. Jorgenson, J. W.; Lukacs, K. D. Science 1983, 222, 266-272.

5. Lauer, H. H.; McManigill, D. Anal. Chem. 1986, 58, 166-170.

6. Grossman, P. D.; Colburn, J. C.; Lauer, H. H.; Nielsen, R. G.; Riggin, R. M.; Sittampalam, G. S.; Rickard, E. C. Anal. Chem. 1989, 61, 1186-1194.

7. Ewing, A. G.; Wallingford, R. A.; Olefirowicz, R. M. Anal. Chem. 1989, 61, 292A-303A.

8. Rose, D. J.; Jorgenson, J. W. J. Chromatogr. 1988, 438, 23-34.

9. Jorgenson, J. W.; Lukacs, K. D. Anal. Chem. 1981, 53, 1298-1302.

10. Lukacs, K. D.; Jorgenson, J. W. J. High Resolut. Chromatogr. Chromatogr. Commun. 1985, 8, 407-411.

11. Altria, K. D.; Simpson, C. F. Anal. Proc. 1986, 23, 453-454.

12. Hjertén, S. J. Chromatogr. 1985, 347, 191-198.

13. Bruin, G. J. M.; Chang, J. P.; Kuhlman, R. H,; Zegers, K.; Kraak, J. C.; Poppe, H. J. Chromatogr. 1989, 471, 429-436.

14. Lee, C. S.; Blanchard, W. C.; Wu, C.-T. Anal. Chem. 1990 , $62,1550-1552$

15. Olivares, J. A.; Nguyen, N. T,; Yonker, C. R.; Smith, R. D. Anal. Chem. 1987, 59, 1230-1232.

16. Smith, R. D.; Barinaga, C. J.; Udseth, H. R. Anal. Chem. 1988, 60, 1948-1952.

17. Andresen, B. D.; Fought, E. R. U.S. Patent No. 4,705,616; Nov. 10, 1987.

18. Lee, E. D.; Mück, W.; Henion, J. D.; Covey, T. R. J. Chromatogr. 1988, 458, 313-321.

19. Caprioli, R. M.; Fan, T.; Cottrell, J. S. Anal. Chem. 1986, 58, 2949-2954. 
20. Minard, R. D.; Chin-Fatt, D.; Curry, P.; Ewing, A. G. Proceedings of the 36 th ASMS Conference on Mass Spectrometry and Allied Topics, San Francisco, CA, June 5-10, 1988, American Society for Mass Spectrometry: East Lansing, MI, 1988; pp 950-951.

21. Moseley, M. A.; Deterding, L. J.; Tomer, K. B.; Jorgenson, J. W. Rapid Commun. Mass Spectrom. 1989, 3, 87-93.
22. Caprioli, R. M.; Moore, W. T.; Martin, M.; DaGue, B. B.; Wilson, K,; Moring, S. J. Chromatogr. 1989, 480, 247-257.

23. Foret, F.; Deml, M.; Boček, P. J. Chromatogr. 1988, 452, 601-613.

24. Grossman, P. D.; Colburn, J. C.; Lauer, H. H. Anal. Biochem. 1989, 179, 28-33. 